\title{
How does the brain sustain a visual percept?
}

\author{
G. M. Portas*, B. A. Strange, K. J. Friston, R. J. Dolan and C. D. Frith \\ Wellcome Department of Cognitive Neurology, Institute of Neurology, 12 Queen Square, London WC1N 3BG, UK
}

\begin{abstract}
Perception involves the processing of sensory stimuli and their translation into conscious experience. A novel percept can, once synthesized, be maintained or discarded from awareness. We used event-related funtional magnetic resonance imaging to separate the neural responses associated with the maintenance of a percept, produced by single-image, random-dot stereograms, from the response evoked at the onset of the percept. The latter was associated with distributed bilateral activation in the posterior thalamus and regions in the occipito-temporal, parietal and frontal cortices. In contrast, sustained perception was associated with activation of the pre-frontal cortex and hippocampus. This observation suggests that sustaining a visual percept involves neuroanatomical systems which are implicated in memory function and which are distinct from those engaged during perceptual synthesis.
\end{abstract}

Keywords: perception; memory; hippocampus; dorsolateral prefrontal cortex

\section{INTRODUCTION}

A conscious experience is a complex phenomenon which is thought to evolve through sequential cognitive steps (Libet 1965). Changes in visual percepts are associated with widespread patterns of brain activation (Kleinshmidt et al. 1998; Lumer et al. 1998). However, little is known about the neural correlates of sustained perceptual states. It is conceivable that a sustained percept is implemented simply by enduring activity in the areas responsible for the initial perceptual synthesis. Alternatively, the maintenance of a percept could be mediated by brain areas distinct from those involved in perceptual transitions. In this event, understanding the neural correlates of perceptual maintenance will be fundamental in understanding the mechanisms associated with visual awareness (Zeki \& Bartels 1999).

We used event-related, functional magnetic resonance imaging (fMRI) to identify the neural responses associated with the onset and maintenance of a visual percept produced by single-image, random-dot stereograms (SIRDSs) in order to test the hypothesis that these perceptual processing stages have distinct neural correlates. Based on previous work we anticipated that the onset of a new percept would be associated with the activation of the occipito-fronto-parietal cortices as suggested by Kleinschmidt et al. (1998) and Lumer et al. (1998). However, there are no data available to suggest how a sustained percept may be implemented in the brain.

\section{METHODS}

The characterization of brain responses mediating conscious perception is confounded by activity attributable to processing the physical properties of a stimulus. To resolve this problem we used visual stimuli (SIRDSs), which, although remaining constant in terms of their physical attributes, produce a definable change in conscious visual perception (Tyler \& Clarke 1990). A SIRDS consists of an intricate pattern of dots in which two similar shapes (separated by an angle of $2-3^{\circ}$ ) are embedded in the background (figure 1). Each eye of an observer

*Author for correspondence (cportas@fil.ion.ucl.ac.uk). is presented with a slightly different image. This creates binocular disparity and the perception of depth where none exists. Initially, subjects perceive a SIRDS as a pattern of dots (surface image) but after a short period of time perception of a three-dimensional (3D) object is attained (a 3D 'pop-out' effect). The ensuing percept can be maintained by trained observers (3D sustained perception). Consequently, using SIRDSs it is possible to dissociate the effects of early visual processing from those produced by the onset and subsequent maintenance of a percept. To avoid bias to particular stimulus attributes we presented SIRDSs with highly variable semantic and structural contents.

Ten healthy volunteers (nine males and one female aged 2434 years with a mean of 29 years) gave informed consent. The day before the experiment these subjects were tested on three SIRDSs (different from the SIRDSs presented during scanning) to ensure that they required more than $10 \mathrm{~s}$ and less than $1 \mathrm{~min}$ to resolve a SIRDS. The former ensured that the haemodynamic response due to the presentation of the image was dissociated from the response due to pop-out and the latter minimized the scanning time. A total of 40 SIRDSs were presented to each subject during two consecutive sessions of $c a .25 \mathrm{~min}$ each. After scanning, the subjects resolved the 40 experimental SIRDSs again, specifying their identity and any difficulty in resolving a particular 3D image during scanning (debriefing questionnaire). Their responses were used to dissociate the pop-out and consequent maintenance of the 3D image from unstable perceptions alternating between the surface 2D image and the 3D image. Unstable perceptions were considered apart in the data analysis (see below).

For each SIRDS presentation, the subjects were required to make a push-button response at the time when pop-out occurred (sudden onset of a 3D percept). This motor response was accompanied by a short 'beep' $(0.5 \mathrm{~s}$ ) (event type 1 ). The subjects were then required to maintain perception of the popped-out image whilst fixating on the centre of the image (epoch of 3D sustained perception) until the appearance of the next SIRDS.

During the epoch of sustained perception, the subjects were asked to press the button once again when they heard a second beep (occurring between 16 and $22 \mathrm{~s}$ after the pop-out) (event type 2). This design enabled a comparison of the responses evoked by the two different types of events and the epoch of 


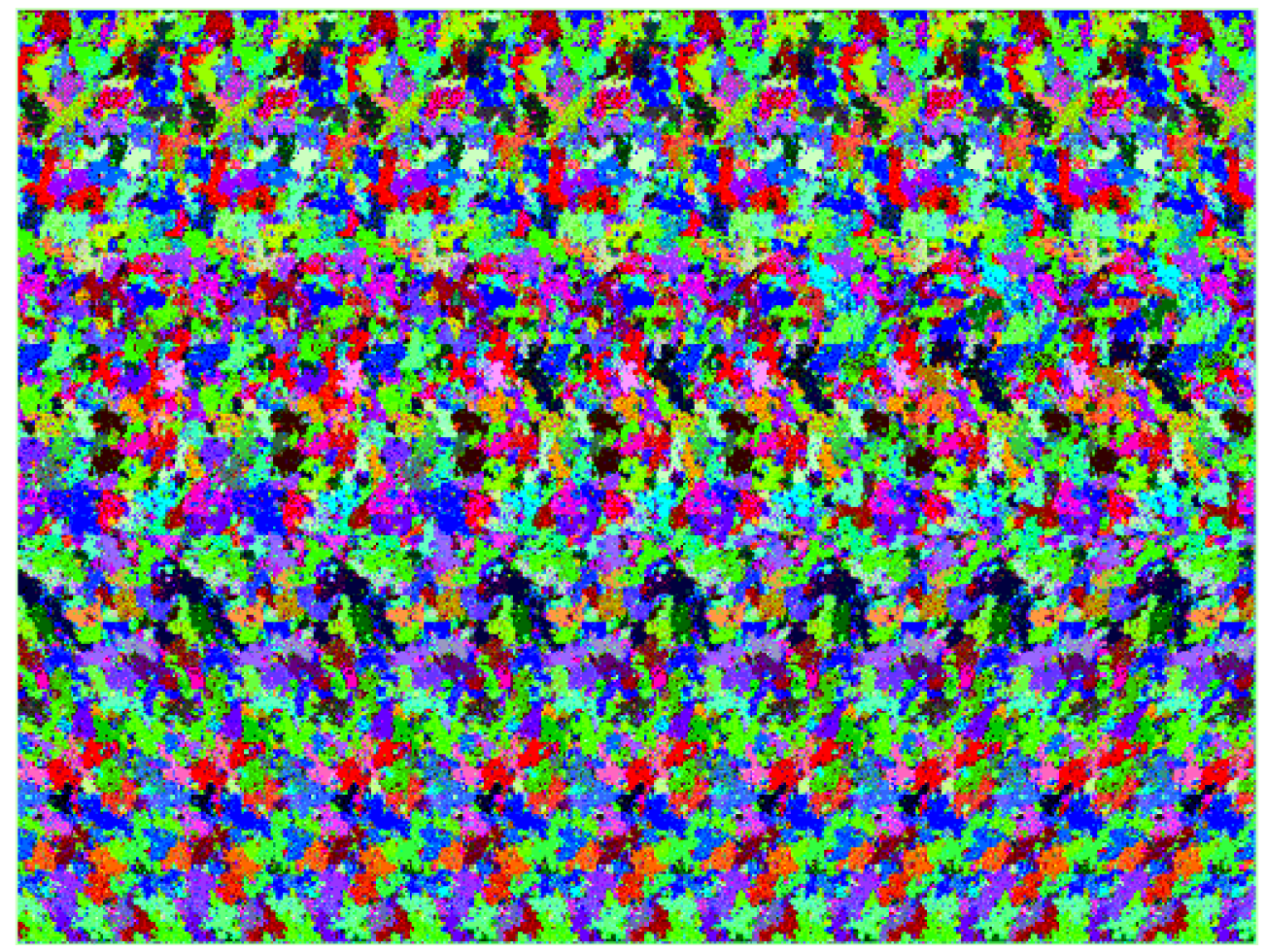

Figure 1. Example of a SIRDS. Note that the surface image appears as a colourful pattern of dots. The pop-out image is a teapot.

sustained perception with baseline activity (figure 2). The epoch of $3 \mathrm{D}$ sustained perception consisted of an unbroken period of perceptual stability and included the two types of events. Event types 1 and 2 shared the same elements, i.e. perception of the same 3D image, hearing the same auditory stimulus and making the same motor response to a stimulus. However, the occurrence of the pop-out effect was exclusive to event type 1 . The events of type 2 were designed to control for the responses that the subjects made in order to indicate when pop-out occurred.

Each cycle (SIRDS presentation plus pop-out plus sustained percept) lasted $c a .60 \mathrm{~s}$. The variability in cycle duration was due to the different times required for perceptual synthesis at each SIRDS presentation (depicted by the dotted line in figure 2). Critically, the visual stimulus was constant over each cycle enabling us to dissociate any effect, due to onset and subsequent maintenance of a percept, from visual processing of the physical attributes of the SIRDS.

Anatomical (Tl-weighted) and functional (T2*-weighted) images were acquired for each subject with a 2 Tesla Magnetom VISION MRI scanner (Siemens, Erlangen, Germany). Contiguous, multislice, echo-planar, $\mathrm{T}^{*}$-weighted image volumes were obtained with blood oxygenation level-dependent (BOLD) contrast using an axial slice orientation and an echo time of $40 \mathrm{~ms}$. The volume acquired covered the whole brain (48 slices of voxel size $3 \mathrm{~mm} \times 3 \mathrm{~mm} \times 3 \mathrm{~mm}$ ) and the field of view was $192 \mathrm{~mm}$. The repetition time between volumes was $4.1 \mathrm{~s}$.

The functional volumes acquired from each subject were realigned (to correct for motion), co-registered with the subject's T1 anatomical image, spatially normalized to a stereotactic space (Talairach \& Tournoux 1988) and smoothed (spatially filtered) using a $6 \mathrm{~mm}$ Gaussian kernel (Friston et al. 1996). Global changes in the BOLD signal were removed by proportional scaling (Holmes et al. 1997).

The data were analysed using statistical parametric mapping (SPM) employing a random effects model (Holmes \& Friston
1998) implemented with a two-level procedure. To test for the effects of interest we used a conventional analytical approach. We specified three effects of interest (figure 2): 'the 3D pop-out effect', 'sustained 3D perception' and 'sensori-motor integration' (the latter modelling the motor responses and beep stimuli in event types 1 and 2). These effects were modelled by convolving delta functions (pop-out and sensori-motor integration) or boxcar functions (sustained perception) with a haemodynamic response function to create three regressors of interest. In some subjects it was necessary to add a fourth effect which modelled perceptions alternating between the surface and 3D images. This effect, as well as low-frequency fluctuations in the signal intensity, were modelled as regressors of no interest. The random effects analysis involved three steps. First, session-specific parameter estimates pertaining to each effect of interest were calculated for each subject for each voxel, producing an image of parameter estimates (Holmes \& Friston 1998). Second, the specific contrasts of the parameter estimates (i.e. the appropriate weights which specify the comparisons between the effects of interest) were calculated in a voxel-wise manner to produce one contrast image per contrast per subject. The three effects modelled (namely the 3D pop-out effect, sustained 3D perception and sensori-motor integration) showed a degree of colinearity. During the contrast image calculation, we removed these correlations by orthogonalizing them with respect to each other (Strange et al. 1999). Finally, the contrast images (one for each subject) for each effect were entered into a one-tailed $t$-test. The set of $T$-values thus obtained constituted a statistical parametric map SPM $\{t\}$ (Friston et al. 1995).

The SPMs for each of the three effects modelled were transformed into $\operatorname{SPM}(Z)$ s. Ensuing areas of activation were characterized in terms of their peak heights ( $\mathcal{Z}$-value maxima) with their positions specified (in coordinates $x, y$ and $z$ ) according to the stereotactic space of Talairach \& Tournoux (1988). We report activations above a threshold corresponding to $p<0.001$ (uncorrected for multiple comparisons) in the areas for which activation had been hypothesized. All other activations 
(a)

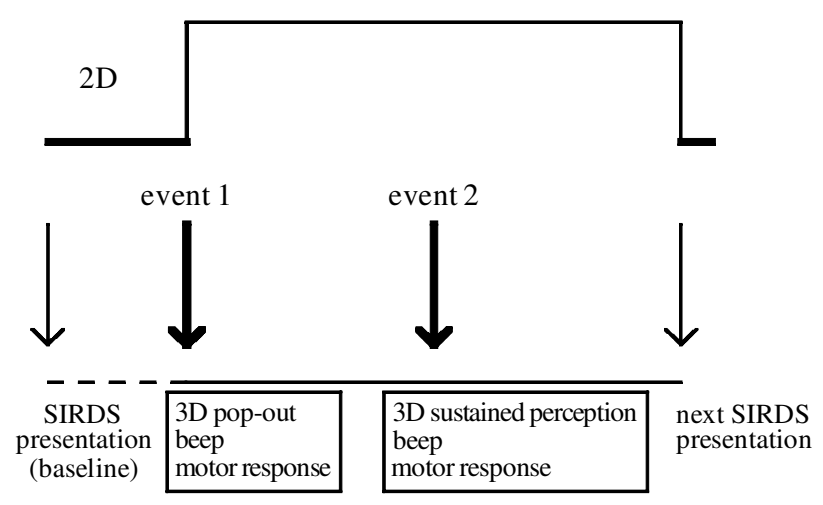

(b)
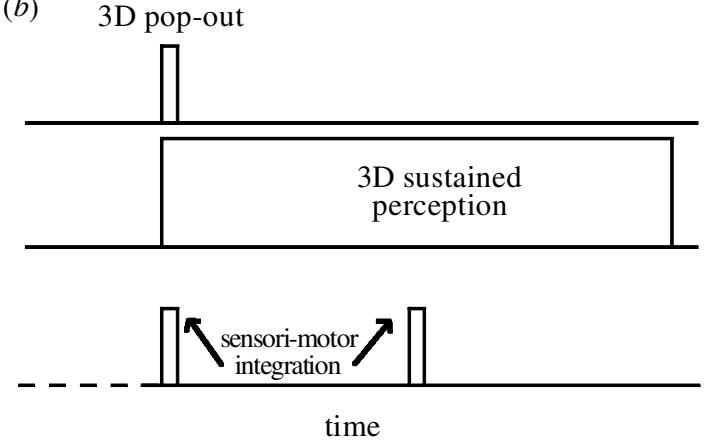

Figure 2. Schematic representation of $(a)$ the experimental design and $(b)$ the modelled effects of interest. The effects of interest $(b)$ were convolved with a haemodynamic response function to provide the regressors used to estimate the parameters of each effect in each subject. Event type 1 consisted of the appearance of the hidden image (3D pop-out effect), a motor response (button press) and hearing an auditory stimulus (beep). Event type 2 was designed to control for the response that subjects made indicating when pop-out occurred, and consisted of hearing an auditory stimulus (beep) and making a motor response to this auditory stimulus. Event types 1 and 2 shared many components (perception of the same 3D image, hearing the same auditory stimulus and making the same motor response to a stimulus) but the pop-out effect was exclusive to event type 1 . The trigger for the motor response is different in the two event types. However, both the variance due to the sensori-motor response as well as any differential activation due to cue-related effects were modelled as sensori-motor integration and did not contribute to the onset and maintenance-evoked estimates (see §2). Finally, the epoch of 3D sustained perception consisted of a continuous period of perceptual stability and encompassed the two types of events.

reported survived a threshold corresponding to $p<0.05$ corrected. This correction employs standard procedures which correct for the multiplicity of voxels and the spatial correlations between them using the theory of random Gaussian fields (Friston et al. 1994).

\section{RESULTS}

Nine subjects met the entry criteria as shown by their responses to the questionnaire. One subject could not complete the experiment since he failed to comply with the task instructions. Thus, data from nine subjects (eight males and one female) are presented in this section as a group analysis. Our results indicated that significant
Table 1. Talairach coordinates $(x, y, z)$ and maxima (Z-score) relating to 'pop-out'

$(\mathrm{sP}$, superior parietal cortex; iT, inferior temporal cortex; iO, inferior occipital cortex; pM, premotor cortex.)

\begin{tabular}{|c|c|c|c|c|c|c|c|c|}
\hline & \multicolumn{4}{|c|}{ left hemisphere } & \multicolumn{4}{|c|}{ right hemisphere } \\
\hline & \multicolumn{3}{|c|}{ coordinates } & \multirow[b]{2}{*}{$Z$-score } & \multicolumn{3}{|c|}{ coordinates } & \multirow[b]{2}{*}{$Z$-score } \\
\hline & $x$ & $y$ & $z$ & & $x$ & $y$ & $z$ & \\
\hline $\mathrm{sP}$ & -26 & -60 & 46 & 3.58 & 30 & -60 & 56 & 3.49 \\
\hline $\mathrm{iT} / \mathrm{iO}$ & -32 & -72 & -14 & 4.47 & 42 & -80 & -10 & 4.79 \\
\hline $\mathrm{iO}$ & -38 & -88 & 8 & 3.98 & 44 & -84 & 6 & 4.49 \\
\hline $\mathrm{pM}$ & -52 & 12 & 36 & 3.37 & 48 & 8 & 30 & 3.85 \\
\hline pulvinar & -14 & -26 & -2 & 3.59 & 14 & -26 & 0 & 3.50 \\
\hline
\end{tabular}

haemodynamic changes were elicited in distinct brain systems by two effects of interest (the $3 \mathrm{D}$ pop-out effect and sustained $3 \mathrm{D}$ perception).

The onset of a percept (3D pop-out) was associated with a bilateral pattern of activation in the superior parietal, extrastriate occipital, inferior temporal and premotor cortices and the pulvinar (table 1 and figure 3). Single-subject analyses of these data revealed a similar and highly consistent pattern of activation (data not shown).

To determine the neuroanatomical correlates of sustained perception, brain activation during 3D sustained perception was compared to baseline activity (i.e. activation during 2D perception was subtracted from 3D perception). Critically, any evoked response that could be explained by 3D pop-out (event-type 1) or sensori-motor integration (event types 1 and 2) was discounted in this comparison (see $\S 2$ ). Sustained 3D perception was associated with significant $(p<0.05$ corrected) activation in two brain regions: the left dorsolateral pre-frontal cortex and left anterior hippocampus (table 2 and figure 4).

\section{DISCUSSION}

Pop-out-related activation in the occipito-frontoparietal regions is largely consistent with the response elicited during perceptual transitions due to binocular rivalry and ambiguous figures (Kleinschmidt et al. 1998; Lumer et al. 1998) (see tables 3-5 for comparison). We argue that the activation of a thalamocortical network, which is associated with the pop-out effect, mainly reflects functional integration among areas mediating object representation (the occipito-temporal cortex (Treisman \& Kanwisher 1998)) and visual attention (the fronto-parietal cortex (Coull \& Nobre 1998; Portas et al. 1998) and pulvinar (Petersen et al. 1985, 1987; La Berge \& Buchsbaum 1990)). Hence, these data suggest a close relationship between the distributed brain systems involved in perceptual synthesis and those mediating selective visual attention (Büchel \& Friston 1997; Büchel et al. 1998). Furthermore, it is possible that changes in brain activity at the onset of a $3 \mathrm{D}$ percept also reflect contingent changes in vergence (Petit \& Haxby 1999) and stereopsis (Savoy et al. 1993). 

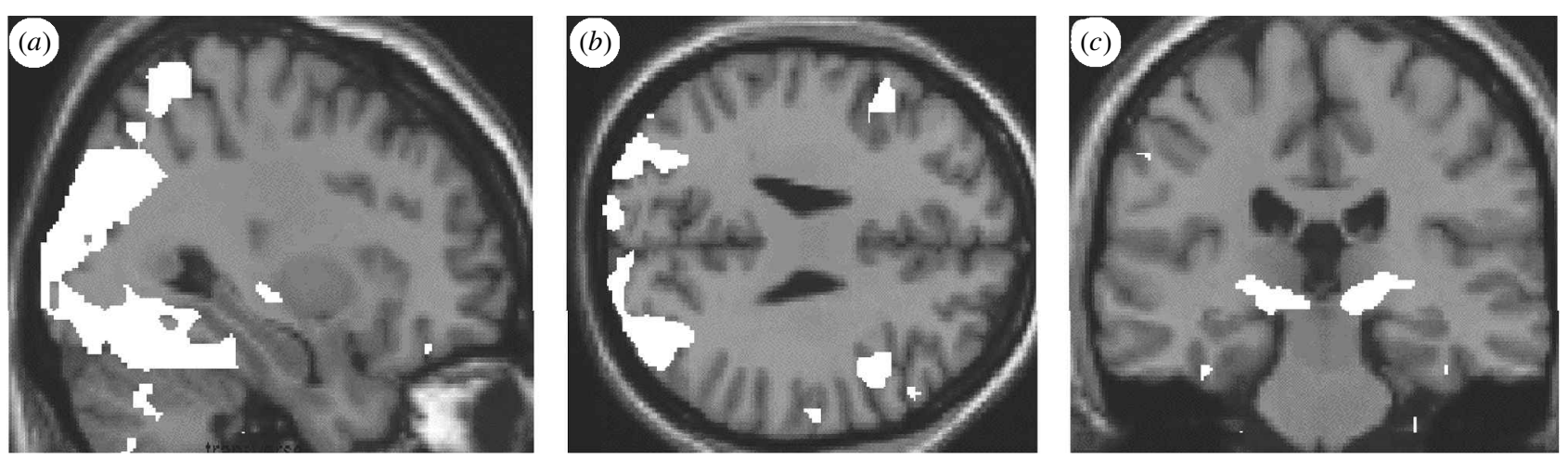

Figure 3. The areas of activation associated with the 3D pop-out effects. (See tables $3-5$ for comparison of our results with previous studies.) The activations were thresholded at $p<0.001$, colour coded and superimposed on a standard T1 template image (Montreal Neurological Institute). Activation in the superior parietal region included a part of the intraparietal sulcus (BA 7/40) (Talairach coordinates $x, y$ and $z: \mathrm{L}-26,-60$ and 56 and $Z$-score 3.58, and $\mathrm{R} 30,-60$ and -56 and $z$-score 3.49 ) (a). This figure also shows an area of activation located between the inferior temporal gyrus and inferior occipital gyrus, including the posterior fusiform gyrus (Talairach coordinates $x, y$ and $z: \mathrm{L}-32,-72$ and -14 and $Z$-score 4.47 , and $\mathrm{R} 42,-80$ and -10 and $Z$-score 4.79$)$ and an area of activation in the inferior occipital gyrus including the inferior bank of the transverse occipital sulcus (Talairach coordinates $x, y$ and $z$ : $\mathrm{L}-38,-88$ and 8 and $Z$-score 3.98 , and $\mathrm{R} 44,-84$ and 6 and $Z$-score 4.49) (BA 19/37). The activation in the pre-motor cortex was located at the intersection between the pre-central sulcus and the inferior frontal sulcus (Talairach coordinates $x, y$ and $z: \mathrm{L}-52,12$ and 36 and $z$-score 3.37, and $\mathrm{R} 48,8$ and 30 and $z$-score 3.85) (BA 6) (b). Activation in the pulvinar is shown in (c). (Talairach coordinates $x, y$ and $z: \mathrm{L}-14,-26$ and -2 and $Z$-score 3.59 , and $\mathrm{R} 14,-26$ and 0 and $Z$-score 3.50$)$.

Table 2. Talairach coordinates $(x, y, z)$ and maxima $(Z$-score $)$ relating to 'sustained perception'

(pF, prefrontal cortex; hip, hippocamus.)

\begin{tabular}{lrrrr}
\hline \multicolumn{4}{c}{ left hemisphere } \\
\cline { 2 - 5 } & \multicolumn{3}{c}{ coordinates } \\
\cline { 2 - 5 } & \multicolumn{1}{c}{$x$} & $y$ & $z$ & z-score \\
\cline { 2 - 5 } & -24 & 26 & 36 & 5.23 \\
$\mathrm{pF}$ & -28 & -10 & -26 & 5.04 \\
\hline
\end{tabular}

The brain activation associated with the maintenance of the percept included the hippocampus and pre-frontal regions. Encoding, retrieval and manipulation of information is known to involve the dorsal regions of the lateral pre-frontal cortex (Petrides et al. 1993a,b, 1995; Henson et al. 1999) and the hippocampus (Gray \& Rawlins 1986; Eichenbaum et al. 1991; Knowlton \& Fanselow 1998).

It is possible that a working memory-like process could be engaged in keeping the perceptual representation active, i.e. preventing it reversing to the surface image. We argue that, once the percept is established, recognition of the percept directs oculomotor function in order to maintain an active representation of that percept. It would be surprising if perceptual synthesis did not engage the oculomotor systems (the pre-motor theory of attention) (Rizzolati 1983). However, the brain areas known to be active during eye movements (Petit \& Haxby 1999) and stereopsis (Savoy et al. 1993) did not activate during perceptual maintenance. We suggest that a tonic top-down control of the oculomotor areas may take place during endurance of a percept by means of

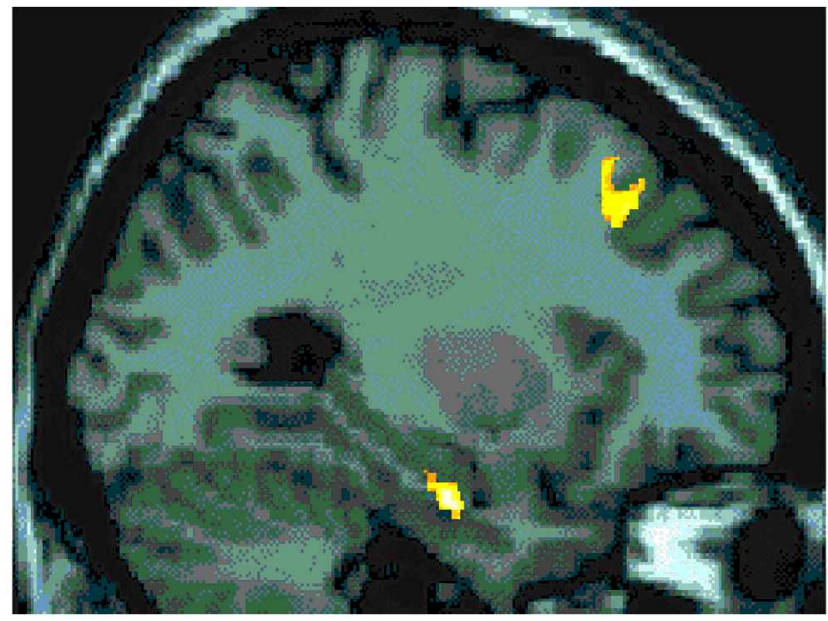

Figure 4. The areas of activation associated with sustained $3 \mathrm{D}$ perception. The figure shows activation of the left dorsolateral pre-frontal cortex (BA 9/46) (Talairach coordinates $x, y$ and $z: \mathrm{L}-24,26$ and 36 and $Z$-score 5.23) and left hippocampus (Talairach coordinates $x, y$ and $z$ : $\mathrm{L}-28,-10$ and -26 and $Z$-score 5.04$)(p<0.05$ corrected $)$.

modulation by higher systems (e.g. the pre-frontal cortex in our study).

Activation of the dorsolateral pre-frontal cortex may reflect this top-down mechanism (Goldman-Rakic 1988; Fuster 1989; Frith et al. 1991; Courtney et al. 1998).

The hippocampus and surrounding medial temporal cortex are critical for long-term memory function which is accessible to conscious recollection and which is referred to as declarative memory (Milner 1972; Squire 1992). The maintenance of a percept places no explicit demand on the declarative memory. However, the hippocampus is thought to mediate relational processing (Eichenbaum 1997) and it may therefore be the case that the maintenance of a conscious percept recruits relational 
Table 3. Comparison of parietal activations across studies, Talairach coordinates $(x, y, z)$ and maxima (Z-score)

\begin{tabular}{|c|c|c|c|c|c|c|c|c|}
\hline \multirow[b]{3}{*}{ reference } & \multicolumn{4}{|c|}{ left hemisphere } & \multicolumn{4}{|c|}{ right hemisphere } \\
\hline & \multicolumn{3}{|c|}{ coordinates } & \multirow[b]{2}{*}{$z$-score } & \multicolumn{3}{|c|}{ coordinates } & \multirow[b]{2}{*}{ Z-score } \\
\hline & $x$ & $y$ & $z$ & & $x$ & $y$ & $z$ & \\
\hline $\begin{array}{l}\text { present } \\
\text { study }\end{array}$ & -26 & -60 & 46 & 3.58 & 30 & -60 & 56 & 3.49 \\
\hline $\begin{array}{l}\text { Kleinschmidt } \\
\quad \text { et al. }\end{array}$ & -18 & -57 & 45 & 4.9 & 21 & -60 & 48 & 4.9 \\
\hline Lumer et al. & - & - & - & - & 30 & -54 & 54 & 7.53 \\
\hline
\end{tabular}

Table 4. Comparison of frontal activations across studies, Talairach coordinates $(x, y, z)$ and maxima (Z-scores)

\begin{tabular}{|c|c|c|c|c|c|c|c|c|}
\hline \multirow[b]{3}{*}{ reference } & \multicolumn{4}{|c|}{ left hemisphere } & \multicolumn{4}{|c|}{ right hemisphere } \\
\hline & \multicolumn{3}{|c|}{ coordinates } & \multirow[b]{2}{*}{$z$-score } & \multicolumn{3}{|c|}{ coordinates } & \multirow[b]{2}{*}{ Z-score } \\
\hline & $x$ & $y$ & $z$ & & $x$ & $y$ & $z$ & \\
\hline $\begin{array}{l}\text { present } \\
\text { study }\end{array}$ & -52 & 12 & 36 & 3.37 & 48 & 8 & 30 & 3.85 \\
\hline $\begin{array}{l}\text { Kleinschmidt } \\
\quad \text { et al. }\end{array}$ & -57 & 12 & 9 & 5 & 48 & 24 & 9 & 4.4 \\
\hline Lumer et al & - & - & - & - & 54 & 15 & 27 & 7.64 \\
\hline Lumer et al. & -57 & 9 & 3 & 7.33 & 60 & 15 & -3 & 8.11 \\
\hline
\end{tabular}

Table 5. Comparison of occipital activations across studies, Talairach coordinates $(x, y, z)$ and maxima $(Z$-score $)$

\begin{tabular}{|c|c|c|c|c|c|c|c|c|}
\hline \multirow[b]{3}{*}{ reference } & \multicolumn{4}{|c|}{ left hemisphere } & \multicolumn{4}{|c|}{ right hemisphere } \\
\hline & \multicolumn{3}{|c|}{ coordinates } & \multirow[b]{2}{*}{$z$-score } & \multicolumn{3}{|c|}{ coordinates } & \multirow[b]{2}{*}{ Z-score } \\
\hline & $x$ & $y$ & $z$ & & $x$ & $y$ & $z$ & \\
\hline $\begin{array}{l}\text { present } \\
\text { study }\end{array}$ & -32 & -72 & -14 & 4.47 & 42 & -80 & -10 & 4.79 \\
\hline $\begin{array}{l}\text { Kleinschmidt } \\
\text { et al. }\end{array}$ & -33 & -54 & -21 & 6.1 & 42 & -51 & -27 & 7.8 \\
\hline Lumer et al. & -30 & -69 & -18 & 7.77 & 33 & -45 & -21 & 8.1 \\
\hline
\end{tabular}

processing during extraction of the structural features constituting the $3 \mathrm{D}$ percept. This suggestion is supported by recent evidence that the medial temporal cortex, along with its critical role in the declarative memory, also mediates perceptual processing (Murray \& Bussey 1999) particularly in the representation of complex conjunctions of visual features (Tanaka 1996). Importantly, hippocampal activation during the maintenance of a percept suggests that such relational processing is automatic, occurring regardless of whether or not an individual is explicitly engaged in encoding. The overlap between perceptual-induced activation and mnemonic function in the hippocampus might further suggest that the maintenance of a percept engages memory-related processing such that a current sensory input is analysed in relation to prior representations (memories or stored knowledge). In other words, the maintenance of a percept is likely to recruit the cognitive processes mediating the interpretation' of the visual sensorium as a result of acquired experience. The hippocampus is not necessary for awareness in general, as hippocampal damage impairs the declarative memory without abolishing consciousness, but it is thought that awareness of a memory is an emergent property of hippocampal function (Eichenbaum 1999). Hippocampal processing may mediate awareness of the on-line percept by integrating it with stored knowledge about the perceived object. The interaction between the hippocampus and pre-frontal cortex during the maintenance of a percept may enable incorporation of these on-line memories into the continuity of perceptual experience.

Finally, we note that our results are consistent with a 'predictive coding' perspective on perceptual synthesis (Rao \& Ballard 1999). Here, perceptual transitions are associated with transient activity reflecting a mismatch between sensory input and a prediction which is mediated by top-down afferents from higher systems. Once the percept is established this error-related activity disappears. However, this is contingent on enduring activity in higher systems (e.g. the dorsolateral pre-frontal cortex and the hippocampus in our study) which provide the prediction.

These data indicate that neurophysiological studies of consciousness should distinguish processes related to perceptual transitions from processes necessary for sustained perceptual experience and emphasize the multilevel nature of the neuronal interactions involved in perception.

This work was supported by grants from the Wellcome Trust. C.M.P. holds a Marie Curie Fellowship. B.A.S. is supported by the Astor Foundation Scholarship. We thank Dr D. Adams and Mr A. Bartels for helpful suggestions and discussion.

\section{REFERENCES}

Büchel, C. \& Friston, K. J. 1997 Modulation of connectivity in visual pathways by attention: cortical interactions evaluated with structural equation modelling and fMRI. Cerebr. Cortex 7 , 768-778.

Büchel, C., Josephs, O., Rees, G., Turner, R., Frith, C. D. \& Friston, K. J. 1998 The functional anatomy of attention to visual motion. A functional MRI study. Brain 121, 1281-1294.

Coull, J. T. \& Nobre, A. C. 1998 Where and when to pay attention: the neural systems for directing attention to spatial locations and to time intervals as revealed by both PET and fMRI. 7. Neurosci. 18, 7426-7435.

Courtney, S. M., Petit, L., Haxby, J. V. \& Ungerleiden, L. G. 1998 The role of prefrontal cortex in working memory: examining the content of consciousness. Phil. Trans. R. Soc. Lond. B 353, 1819-1828.

Eichenbaum, H. 1997 Declarative memory: insights from cognitive neurobiology. A. Rev. Psychol. 48, 547-572.

Eichenbaum, H. 1999 Conscious awareness, memory and the hippocampus. Nature Neurosci. 2, 775-776.

Eichenbaum, H., Cohen, N. J., Otto, T. \& Wible, C. 1991 Memory representation in the hippocampus: functional domain and functional organization. In Memory: organization and locus of change (ed. L. R. Squire, N. M. Weinberger, G. Lynch \& J. L. McGaugh), pp. 163-204. Oxford University Press. 
Friston, K. J., Worsley, K. J., Frackowiak, R. S. J., Mazziotta, J. C. \& Evans, A. C. 1994 Assessing the significance of focal activation using their spacial extent. Hum. Brain Mapp. 1, 214-220.

Friston, K. J., Holmes, A. P., Worsley, K. J., Poline, J. B., Frith, C. D. \& Frackowiak R. S. J. 1995 Statistical parametric mapping in functional imaging: a general linear approach. Hum. Brain Mapp. 2, 189-210.

Friston, K. J., Ashburner, J., Poline, J. B., Frith, C. D., Heather, J. D. \& Frackowiak, R. S. J. 1996 Spatial realignment and normalization of images. Hum. Brain Mapp. 3, 165-189.

Frith, C. D., Friston, K. J., Liddle, P. F. \& Frackowiak, R. J. S. 1991 Willed action and the prefrontal cortex in man: a study with PET. Proc. R. Soc. Lond. B 244, 241-246.

Fuster, J. M. 1989 Defective planning. In The prefrontal cortex. Anatomy, physiology, and neuropsychology of the frontal lobe (ed. J. M. Foster), pp. 138-140. New York: Raven Press.

Goldman-Rakic, P. S. 1988 Topography of cognition: parallel distributed networks in primate association cortex. A. Rev. Neurosci. 11, 137-156.

Gray, J. A. \& Rawlins, J. N. P. 1986 Comparator and buffer memory: an attempt to integrate two models of hippocampal function. In The hippocampus, vol. 4 (ed. R. L. Isaacson \& K. H. Pribram), pp. 159-202. New York: Plenum Press.

Henson, R., Rugg, M. D., Shallice, T., Josephs, O. \& Dolan, R. J 1999 Recollection and familiarity in recognition memory. $\mathcal{F}$. Neurosci. 19, 3962-3972.

Holmes, A. P. \& Friston, K. J. 1998 Generalisability, random effects and population inference. Neuroimage 7, S754.

Holmes, A. P., Josephs, O., Büchel, C. \& Friston, K. J. 1997 Statistical modelling of low-frequency confounds in fMRI. Neuroimage 5, S480.

Kleinschmidt, A., Büchel, G., Zeki, S. \& Frackowiak, R. S. J. 1998 Human brain activity during spontaneously reversing perception of ambiguous pictures. Proc. R. Soc. Lond. B 265, 2427-2433.

Knowlton, B. J. \& Fanselow, M. S. 1998 The hippocampus, consolidation and on-line memory. Curr. Opin. Neurobiol. 8, 293-296.

La Berge, D. \& Buchsbaum, M. S. 1990 Positron emission tomographic measurements of pulvinar activity during an attention task. 7. Neurosci. 10, 613-619.

Libet, B. J. 1965 Brain stimulation and the threshold of conscious experience. In Brain and conscious experience (ed. J. C. Eccles), pp. 165-181. Berlin: Springer.

Lumer, E. D., Friston, K. J. \& Rees, G. 1998 Neural correlates of perceptual rivalry in the human brain. Science $\mathbf{2 8 0}$, 1930-1934.

Milner, B. 1972 Disorders of learning and memory after temporal lobe lesions in man. Clin. Neurosurg. 19, 421-446.

Murray, E. A. \& Bussey, T. J. 1999 Perceptual-mnemonic functions of the perirhinal cortex. Trends Cogn. Sci. 3, 142-151.
Petersen, S. E., Robinson, D. L. \& Keys, W. 1985 Pulvinar nuclei of the behaving rhesus monkey: visual responses and their modulation. F. Neurophysiol. 54, 867-886.

Petersen, S. E., Robinson, D. L. \& Morris, J. D. 1987 Contributions of the pulvinar to visual spatial attention. Neuropsychology 25, 97-105.

Petit, L. \& Haxby, J. V. 1999 Functional anatomy of pursuit eye movements as revealed by fMRI. F. Neurophysiol. 82, 463-471.

Petrides, M., Alivisatos, B., Evans, A. C. \& Meyer, E. $1993 a$ Dissociation of human mid-dorsolateral from posterior dorsolateral frontal cortex in memory processing. Proc. Natl Acad. Sci. USA 90, 873-877.

Petrides, M., Alivisatos, B., Evans, A. C. \& Meyer, E. $1993 b$ Functional activation of the human frontal cortex during the performance of verbal working memory tasks. Proc. Natl Acad. Sci. USA 90, 878-882.

Petrides, M., Alivisatos, B. \& Evans, A. C. 1995 Functional activation of the human ventrolateral frontal cortex during mnemonic retrieval of verbal information. Proc. Natl Acad. Sci. USA 92, 5803-5807.

Portas, C. M., Rees, G., Howseman, A., Josephs, O., Turner, R. \& Frith, C. D. 1998 A specific role for the thalamus in mediating the interaction of attention and arousal in humans. 7. Neurosci. 18, 8979-8999.

Rao, R. P. N. \& Ballard, D. H. 1999 Predictive coding in the visual cortex: a functional interpretation of some extra-classical receptive fields effects. Nature Neurosci. 2, 79-87.

Rizzolati, G. 1983 Mechanism of selective attention in mammals. In Advances in vertebrate neuroethology (ed. J. P. Ewart, R. R. Capranica \& D. J. Ingle), pp. 261-297. New York: Plenum Press.

Savoy, R. L., Kwong, K. K. \& Cohen, M. S. 1993 Searching for stereopsis in humans using ultrafast functional MRI: stimuli, analysis techniques, and preliminary. Soc. Neurosci. Abstr. 19, 1500.

Squire, L. R. 1992 Memory and the hippocampus: a synthesis from findings with rats, monkeys, and humans. Psychol. Rev. 99, 195-231.

Strange, B. A., Portas, C. M., Dolan, R. J., Holmes, A. P. \& Friston, K. J. 1999 Random effects analyses for event-related fMRI. Neuroimage $\mathbf{9}, 36$.

Talairach, P. \& Tournoux, J. 1988 Co-planar stereotaxic atlas of the human brain. New York: Thieme.

Tanaka, K. 1996 Inferotemporal cortex and object vision A. Rev. Neurosci. 19, 109-139.

Treisman, A. M. \& Kanwisher, N. G. 1998 Perceiving visually presented objects: recognition, awareness and modularity. Curr. Opin. Neurobiol. 8, 218-226.

Tyler, C. W. \& Clarke, M. B. 1990 The autostereogram. Proc. Soc. Photo. Illuminat. Eng. 1256, 182.

Zeki, S. \& Bartels, A. 1999 Toward a theory of visual consciousness. Conscious. Cognit. 8, 225-259. 